\title{
Photoelectric effect on dust grains across the L1721 cloud in the $\rho$ Ophiuchi molecular complex ${ }^{\star}$
}

\author{
E. Habart, L. Verstraete, F. Boulanger, G. Pineau des Forêts, F. Le Peintre, and J. P. Bernard \\ Institut d'Astrophysique Spatiale, Bât. 121, Université Paris-Sud, 91405 Orsay Cedex, France
}

Received 15 January 2001 / Accepted 8 March 2001

\begin{abstract}
We present ISO-LWS measurements of the main gas cooling lines, $\left[\mathrm{C}^{+}\right] 158 \mu \mathrm{m}$ and $\left[\mathrm{O}^{0}\right] 63 \mu \mathrm{m}$ towards a moderate opacity molecular cloud $\left(A_{v} \sim 3\right)$, L1721, illuminated by the B2 star $\nu$ Sco $(\chi=5-10)$. These data are combined with an extinction map and IRAS dust emission images to test our understanding of gas heating and cooling in photodissociation regions (PDRs). This nearby PDR is spatially resolved in the IRAS images; variations in the IRAS colors across the cloud indicate an enhanced abundance of small dust grains within the PDR. A spatial correlation between the gas cooling lines and the infrared emission from small dust grains illustrates the dominant role of small dust grains in the gas heating through the photoelectric effect. The photoelectric efficiency, determined from the observations by ratioing the power radiated by gas and small dust grains, is in the range 2 to $3 \%$, in close agreement with recent theoretical estimates (Bakes \& Tielens 1994; Weingartner \& Draine 2001). The brightness profiles across the PDR in the $\left[\mathrm{C}^{+}\right] 158 \mu \mathrm{m}$ and $\left[\mathrm{O}^{0}\right] 63 \mu \mathrm{m}$ lines are compared with model calculations where the density profile is constrained by the extinction data and where the gas chemical and thermal balances are solved at each position. We show that abundance variations of small dust grains across the PDR must be considered to account for the LWS observations.
\end{abstract}

Key words. ISM: clouds - ISM: dust, extinction - atomic processes - molecular processes - radiative transfer

\section{Introduction}

The bulk of interstellar matter is found in regions of low to moderate opacity to UV and visible light where stellar radiation plays a dominant role in determining the chemical and thermal state of the gas. These photondominated or photodissociation regions have been the subject of many observations and theoretical studies over the past twenty years (for a review see Hollenbach \& Tielens 1999). In the classical theoretical paradigm, a PDR is characterized by its proton density, $n_{\mathrm{H}}$, and a scaling factor, $\chi$, which normalises the incident radiation field to the Solar Neighbourhood radiation field in the far-ultraviolet. Comparison between observations and model calculations have concentrated on bright objects with high $\chi>10^{3}$ and gas density $n_{\mathrm{H}}>10^{3} \mathrm{~cm}^{-3}$ which are the easiest to observe. The gain in sensitivity provided by the Infrared Space Observatory (ISO) for gas line observations now permits one to extend these studies to less excited PDRs with lower $n_{\mathrm{H}}$ and $\chi$, which are the most widespread in

Send offprint requests to: E. Habart,

e-mail: emilie.habart@ias.u-psud.fr

* Based on observations with ISO, an ESA project with instruments funded by ESA Member States (especially the PI countries: France, Germany, The Netherlands and the UK) and with the participation of ISAS and NASA. the interstellar medium (ISM). Models show that in such PDRs the gas heating is dominated by the photoelectric effect on dust grains (Hollenbach et al. 1991; Le Bourlot et al. 1993; Kemper et al. 1999). Photoelectric heating has been the subject of recent theoretical investigations which provide an estimate of the photoelectric yield as a function of grain size where the small grains (radius $\leq 100 \AA$ ) dominate the overall photoelectric heating (Bakers \& Tielens 1994; Weingartner \& Draine 2001). It is thus in low excitation PDRs, including the diffuse interstellar medium, that one can most directly validate this theoretical understanding of a key physical process for the ISM, independently of other heating processes. Recently, Wolfire et al. (1995) have used the work of Bakes \& Tielens (1994, hereafter BT) to compute the equilibrium thermal states of the diffuse atomic medium. For the Solar Neighbourhood, their calculations give a net estimate of the $\left[\mathrm{C}^{+}\right] 158 \mu \mathrm{m}$ line emission per hydrogen atom in agreement with the value derived from the comparison of the FIRAS and H I data at high Galactic latitude (Boulanger et al. 1996a). However, this validation of the BT work depends on the relative gas mass in the cold and warm phases of the ISM which is poorly constrained observationally.

In this paper, we investigate the photoelectric heating of the ISM through the study of a specific, low-excitation 
PDR. The comparison of the power radiated by gas and dust allows us to correlate the gas and dust emission and determine the photoelectric efficiency.

To carry out this study, we selected the Lynds Dark Nebula 1721 (L1721, Lynds 1962), a nearby molecular cloud in the $\rho$ Ophiuchi region. This cloud, as seen in the IRAS data, has a roughly spherical geometry and a moderate opacity to stellar light $\left(A_{v}=3\right.$ at cloud center). It is heated on one side by the B2 star $\nu$ Sco and by a more isotropic interstellar radiation field created by the Upper Scorpius association. We present observations in this paper of the $\left[\mathrm{C}^{+}\right] 158 \mu \mathrm{m}$ and $\left[\mathrm{O}^{0}\right] 63 \mu \mathrm{m}$ lines obtained with the Long Wavelength Spectrometer (LWS, Clegg et al. 1996) on board the Infrared Space Observatory (ISO, Kessler et al. 1996). These observations are combined with IRAS images and an extinction map (Sects. 2-3). Thanks to its proximity to the Sun and the moderate gas density, the L1721 PDR is spatially resolved by both the gas and dust observations. The data is interpreted within the framework of a model of the gas emission (Sect. 3). The data allows us to determine the photoelectric efficiency and to illustrate the dominant role of small dust grains in the photoelectic heating (Sect. 4). The paper conclusions are summarised in Sect. 5 .

\section{Observations}

The L1721 cloud is centered at $\alpha=16 \mathrm{~h} 14 \mathrm{mn} 30.41 \mathrm{~s}$ and $\beta=-18^{\circ} 58^{\prime} 7.9^{\prime \prime}$ (Epoch 2000), at the north of the $\rho$ Ophiuchi complex. The B2 star $\nu$ Sco is located at a distance of $\sim 134 \mathrm{pc}$. The projected distance between the center of the cloud and the star is approximately $1.7 \mathrm{pc}$. We discuss in detail the incident radiation field of L1721 in Sect. 3.2.

On the map of the $12 / 100 \mu \mathrm{m}$ IRAS band ratio (see Fig. 1), the limb brightening of the cloud is clearly visible. Former studies have shown that ratios of the different IRAS bands mostly trace dust abundance variations (Boulanger et al. 1990; Bernard et al. 1993). We discuss the abundance variations of small grains across L1721 in Sect. 3.5.

\subsection{Gas cooling lines}

The $\left[\mathrm{O}^{0}\right] 63 \mu \mathrm{m}$ and $\left[\mathrm{C}^{+}\right] 158 \mu \mathrm{m}$ lines have been observed by ISO with the LWS (TDT $=45401125$, LWS02 grating mode, Clegg et al. 1996). At each sky position, the $\mathrm{C}^{+}$and $\mathrm{O}^{0}$ lines have been observed 8 and 30 times respectively with 5 seconds-individual scans (the integration per grating point was $0.4 \mathrm{~s})$. The resolution $(F W H M)$ is $\Delta \lambda=0.5$ and $0.29 \mu \mathrm{m}$ for the $\mathrm{C}^{+}$and $\mathrm{O}^{0}$ lines respectively and each resolution element was sampled twice. We use the LWS pipeline version 7 for standard data reduction. To remove glitches (cosmic ray impacts on the detectors), we co-add all scans to obtain a first estimate of the mean line spectrum. Subtracting this mean spectrum from each individual scan, we obtain a (noise + glitch)-spectrum in which glitches are identified as $3 \sigma$-and $5 \sigma$-outliers for $\mathrm{C}^{+}$

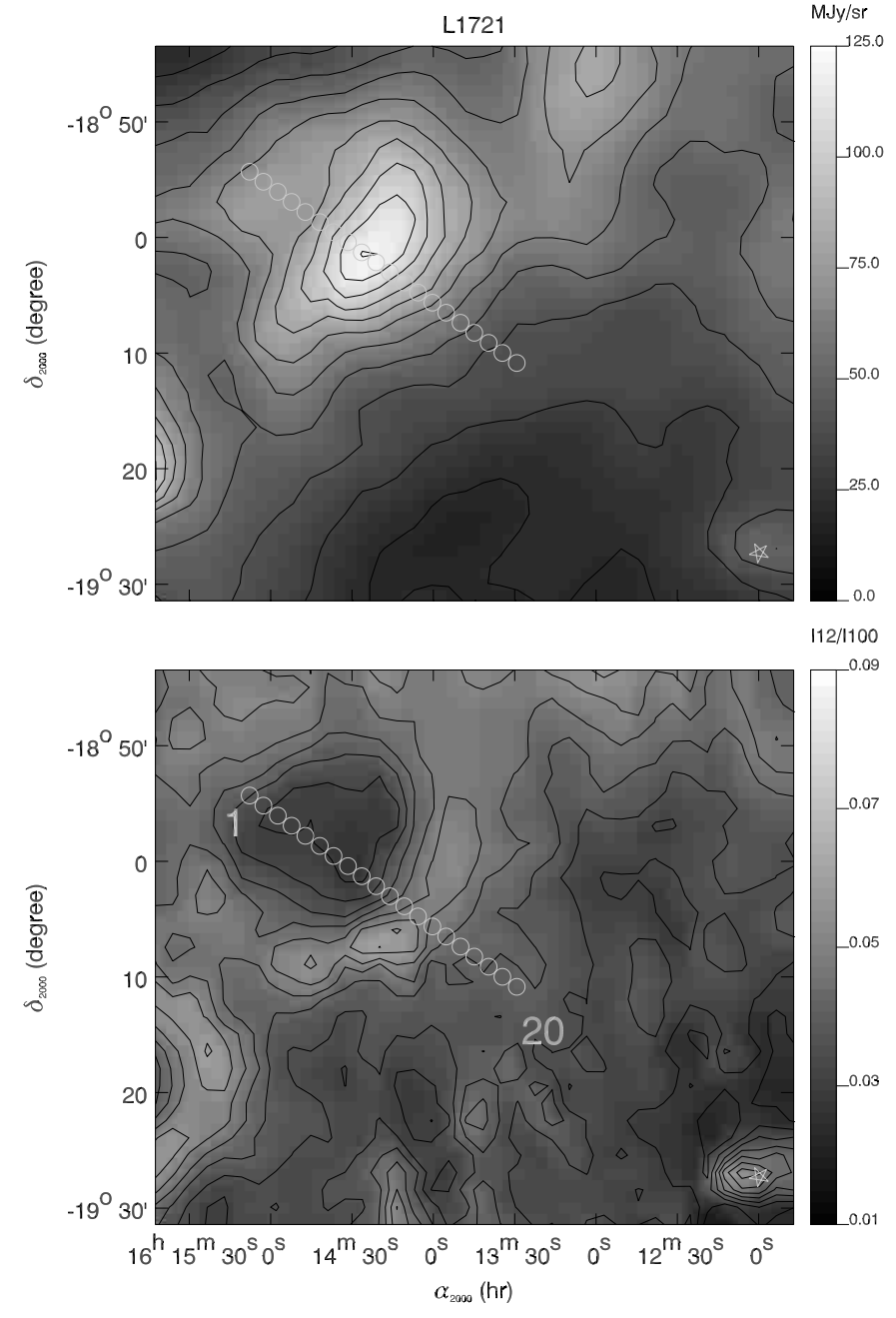

Fig. 1. L1721 as seen in the IRAS data. Top: map of the sum of IRAS bands $(12+25+60+100 \mu \mathrm{m})$ with the beams of LWS observations projected on sky. Bottom: map of the 12/100 $\mu \mathrm{m}$ IRAS band ratio. In this latter map, the limb brightening or halo of the cloud is clearly visible. The circles show the LWS beam in the cut taken across the cloud (20 positions) and the position of $\nu$ Sco is marked by a star.

and $\mathrm{O}^{0}$ respectively. After rejection of glitches, all scans are again co-added to produce the final line spectrum. Line fluxes and error bars are obtained by fitting a Gaussian and a linear baseline to the profiles. Figure 2 shows the line intensity profiles.

To measure the line emission from L1721, we need to subtract the contribution of the diffuse ISM along the line of sight. We express the background contribution to the line emission as:

$I_{b}=Y \times \nu I_{\nu}$

where $Y$ is the line-to-continuum ratio and $\nu I_{\nu}$ the continuum brightness at the line wavelength. In the case of the $\mathrm{C}^{+}$line, we take the line-to-continuum ratio measured in the Galaxy at high latitude, $Y_{\mathrm{C}^{+}}=0.009 \pm 0.0015$ from Boulanger et al. (1996a). This value agrees well with the Galactic plane mean value of 0.0082 reported by Wright et al. (1991) based on the FIRAS (Far-Infrared Absolute 


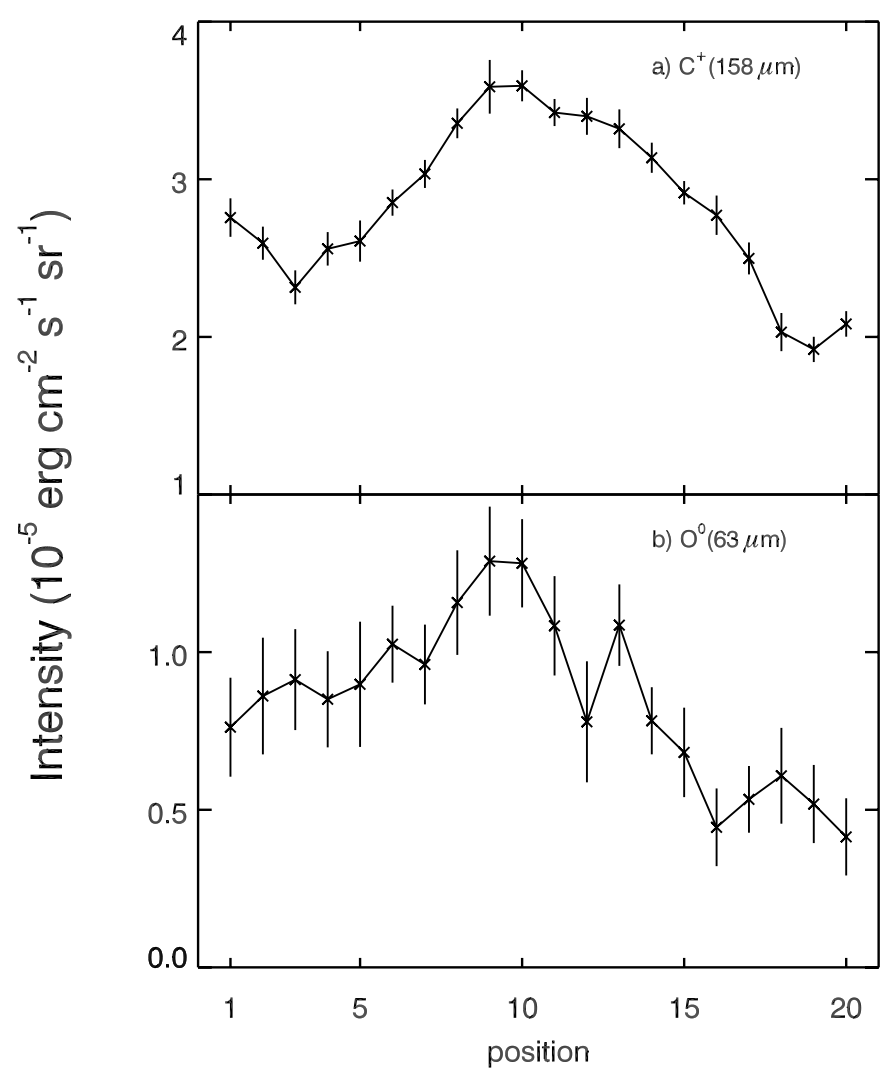

Fig. 2. Intensities of the $\mathrm{C}^{+}$and $\mathrm{O}^{0}$ lines with $3 \sigma$ error bars as measured by LWS at each position across L1721. The background contribution has been subtracted (see text). The $\nu$ Sco star lies at the right end of the cut.

Spectrophotometer) survey of the far-infrared emission from our Galaxy. Wright et al. (1991) observed, as expected theoretically, that the intensity of the $\mathrm{C}^{+}$line scales linearly with the dust continuum emission. The variations of the $\mathrm{C}^{+}$line-to-continuum ratio, due to differences in the dust properties, in the color of the ambient interstellar radiation field or the saturation of the $\mathrm{C}^{+}$cooling rate when the level populations are in equilibrium with dense warm gas, are within $25 \%$ in the plane of Galaxy. In the following we consider that $Y_{\mathrm{C}^{+}}=9 \pm 2.25 \times 10^{-3}$ in the proximity of the L1721 cloud. For the $\mathrm{O}^{0}$ line, the combination of the observations of Caux \& Gry (1997) in the diffuse ISM and the IRAS band at $60 \mu \mathrm{m}$ provide a large range of $Y_{\mathrm{O}^{0}}$ values from $5 \times 10^{-4}$ to $5 \times 10^{-3}$. To derive the $\mathrm{O}^{0}$ background around the L1721 cloud we take the median of these values $Y_{\mathrm{O}^{0}}=1.7 \times 10^{-3}$. Note however that the large uncertainty on $Y_{\mathrm{O}^{0}}$ will not affect our estimate of the $\mathrm{O}^{0}$ line emission in the L1721 cloud because the contribution of the diffuse ISM to the $\mathrm{O}^{0}$ emission along the line of sight is much lower than the line intensity in the cloud. For the continuum brightness, we use the DIRBE (Diffuse Infrared Background Experiment) data corrected for zodiacal light. The continuum values are found from the average of DIRBE points in the diffuse ISM around the L1721 cloud. The variations of the DIRBE values are typically within $20 \%$ in the surrounding of the cloud. We find: $\nu I_{\nu}(158 \mu \mathrm{m})=9.2 \pm 1.9 \times 10^{-4} \mathrm{ergs} / \mathrm{s} / \mathrm{cm}^{2} / \mathrm{sr}$ and $\nu I_{\nu}(60 \mu \mathrm{m})=3.4 \pm 0.7 \times 10^{-4} \mathrm{ergs} / \mathrm{s} / \mathrm{cm}^{2} / \mathrm{sr}$. The corresponding background emission values which have been subtracted from our LWS cut are $8.3 \pm 2.7 \times 10^{-6}$ and $5.8 \pm 1.2 \times 10^{-7} \mathrm{ergs} / \mathrm{s} / \mathrm{cm}^{2} / \mathrm{sr}$ for $\mathrm{C}^{+}$and $\mathrm{O}^{0}$ respectively.
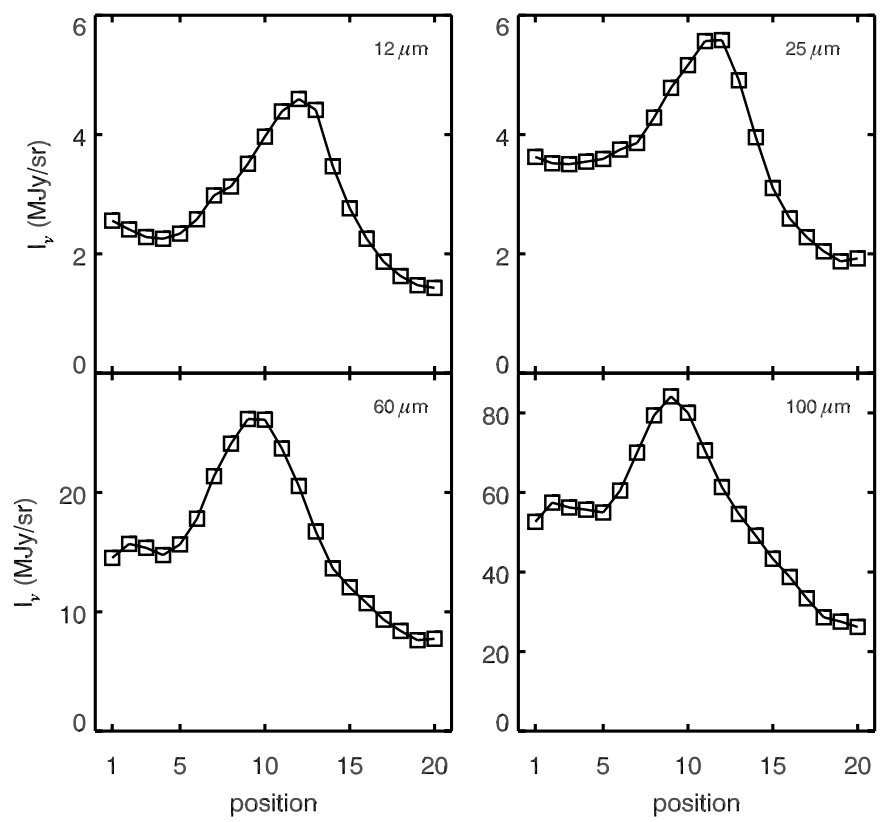

Fig. 3. Surface brightness in the 4 IRAS bands as a function of the observed LWS positions. The background emission has been subtracted (see Sect. 2.2).

\subsection{Dust infrared emission}

Figure 3 shows the background subtracted surface brightness in the four IRAS bands. For each IRAS band, the background emission has been estimated along cuts extending the LWS cut at both ends of the cloud. The background emission is taken to be the average of the minimum values of these extended cuts: $I_{12}^{\mathrm{b}}=1.2 \pm 0.2, I_{25}^{\mathrm{b}}=$ $1.8 \pm 0.3, I_{60}^{\mathrm{b}}=7 \pm 1.6$ and $I_{100}^{\mathrm{b}}=35 \pm 3.9 \mathrm{MJy} / \mathrm{sr}$.

The IRAS and COBE data are well explained with dust models comprising three components (Désert et al. 1990; Dwek et al. 1997). We adopt the Désert et al. model: the three dust components are thus by order of increasing sizes (in terms of the grain radius, $a$ ):

1. large aromatic molecules or PAHs (Polycyclic Aromatic Hydrocarbons) which contribute most of the $12 \mu \mathrm{m}$ band ( $a=4$ to $12 \AA$ );

2. the Very Small Grains (VSGs) which dominate the $25 \mu \mathrm{m}$ and $60 \mu \mathrm{m}$ bands $(a=12$ to $150 \AA)$;

3. the Big Grains (BGs) which make most of the $100 \mu \mathrm{m}$ band $\left(a=1.5 \times 10^{-2}\right.$ to $\left.0.11 \mu \mathrm{m}\right)$.

In the following, we will often refer to PAHs and VSGs as the small dust grain populations. To derive the photoelectric efficiency for each grain component, we need to 
decompose the total infrared (IR) emission into individual dust contributions at each position in the cloud.

In the case of PAHs, we use the following relationship based on ISOCAM and DIRBE data (Boulanger et al. 1996b; Bernard et al. 1994):

$I_{\mathrm{PAH}}=1.5 \times \nu I_{\nu}(12 \mu \mathrm{m}) \mathrm{erg} \mathrm{cm}^{-2} \mathrm{~s}^{-1} \mathrm{sr}^{-1}$.

The emission of big grains is modelled with a modified blackbody of temperature $T_{\mathrm{BG}}$ and an emissivity law proportional to $\nu^{2}$. The value of $T_{\mathrm{BG}}$ is estimated using the flux measured by all ten LWS detectors covering the range $\lambda=42.5-176 \mu \mathrm{m}$. At each position, we fit the ten spectral points with the modified blackbody: a constant temperature of $T_{\mathrm{BG}}=20 \mathrm{~K}$ at all positions is found to provide a satisfactory fit. Finally, this modified blackbody is scaled to match the $100 \mu \mathrm{m}$ IRAS band.

For the VSGs emission, we first obtain two spectral points at 25 and $60 \mu \mathrm{m}$ from:

$I_{25,60}(\mathrm{VSG})=I_{25,60}(\operatorname{IRA} S)-I_{25,60}(\mathrm{BG})$.

At each cloud position, we fit these two points with a modified blackbody of constant temperature $T_{\mathrm{VSG}}=70 \mathrm{~K}$ and an emissivity law proportional to $\nu$.

The bolometric intensity of each dust component is shown in Fig. 4: in fact, the VSGs and BGs emission profiles are quite similar to the $25+60 \mu \mathrm{m}$ and $100 \mu \mathrm{m}$ IRAS band profiles respectively. We note that BGs and VSGs temperatures are expected to decrease as one penetrates into the cloud. The constant temperature we find may be due to the spherical geometry of the cloud where warm and cold dust are mixed along a given line of sight.

\section{Modelling the gas line cooling}

To study the photoelectric effect on dust grains across this nearby PDR, we will compare the total gas cooling lines to the dust IR emission (see Sect. 4). In the case of low excitation PDRs, cooling by the pure rotational lines of $\mathrm{H}_{2}$ may be important. As this latter has not been observed, we resort to an updated version of the PDR model described in Le Bourlot et al. (1993) to estimate the cooling by $\mathrm{H}_{2}$. Moreover the comparison between the brightness profiles observed across the L1721 PDR in the $\left[\mathrm{C}^{+}\right] 158 \mu \mathrm{m}$ and $\left[\mathrm{O}^{0}\right] 63 \mu \mathrm{m}$ lines with model calculations provides a test for our understanding of gas heating/cooling in PDRs. The key ingredients of this model are the density profile across the PDR and the incident radiation field at the cloud surface. In the following, we describe how these quantities are constrained in order to produce a realistic model of the L1721 PDR.

\subsection{The density distribution}

To determine the density profile of L1721 we use a visual extinction map derived from star counts (Cambrésy 1999). Figure 5 displays the column density, $N_{\mathrm{H}}=A_{v} /\left(0.54 \times 10^{-21}\right) \mathrm{cm}^{-2}$ observed across L1721.

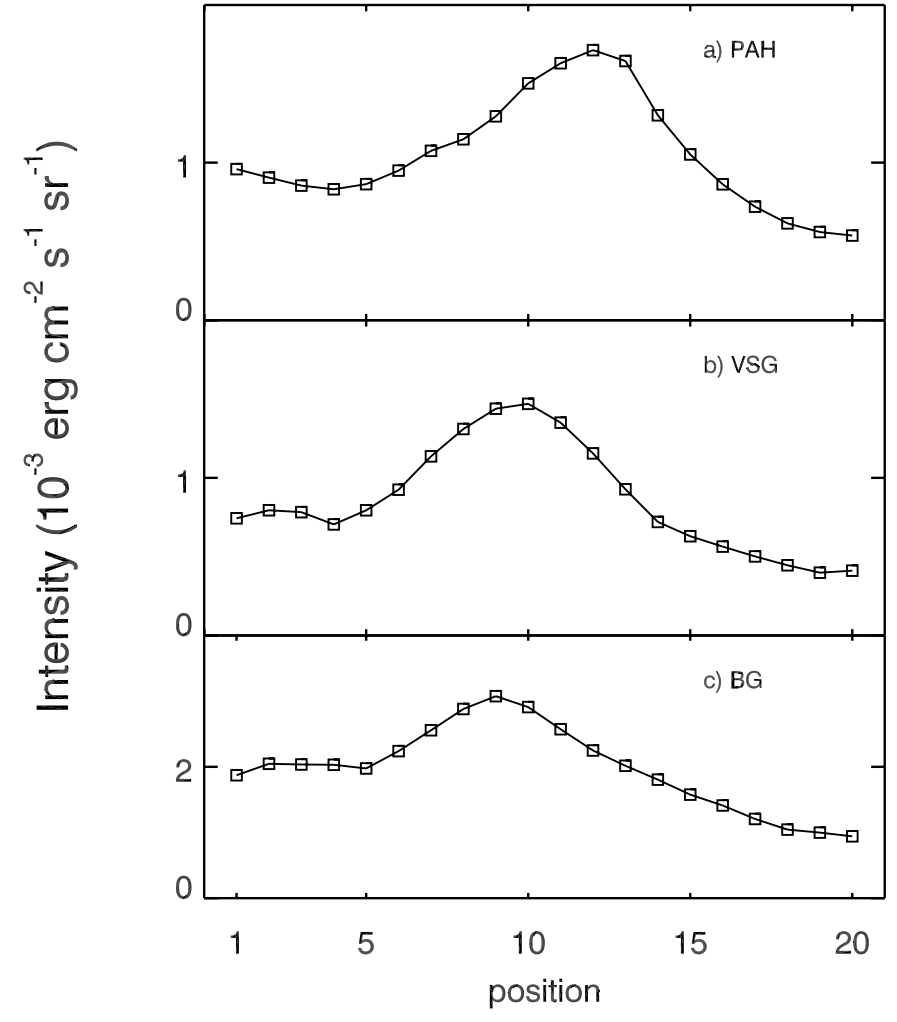

Fig. 4. Integrated flux of each dust component as a function of the position along the LWS cut. $\nu$ Sco lies at the right end of the cut.

The column density profile is well represented by a gas density distribution of the form (see Fig. 5):

$n_{\mathrm{H}}(r)= \begin{cases}n_{\mathrm{H}}^{\mathrm{o}} \times\left(r / r_{1}\right)^{-1.9} & : r_{1}<r \leq r_{m} \\ n_{\mathrm{H}}^{\mathrm{O}} & : \quad r \leq r_{1}\end{cases}$

with $r$ the radial distance to the cloud center, $n_{\mathrm{H}}^{\mathrm{o}}=$ $2915 \mathrm{~cm}^{-3}$ the constant density in the $0 \leq r \leq r_{1}$ region with $r_{1}=0.2 \mathrm{pc}$ and $r_{m}=2.2 \mathrm{pc}$. In this derivation, we assume that $R_{v}$ is constant and equal to 3.1 across the L1721 cloud. A higher value of $R_{v}, 5.5$, that is commonly observed in opaque cores, would multiply our extinction values by a small factor, about 1.12 .

\subsection{The radiation field}

The ratio $I_{100 \mu \mathrm{m}} / N_{\mathrm{H}}$ provides a good measure of the radiation field strength (see Fig. 9 in Bernard et al. 1992). In Fig. 6, we show the relationship between the IRAS $100 \mu \mathrm{m}$ surface brightness and the observed column density. In the diffuse part of the cloud $\left(A_{v} \leq 0.8\right)$, we observe a good linear correlation between the IRAS $100 \mu \mathrm{m}$ surface brightness and the column density. The slope $I_{100 \mu \mathrm{m}} / N_{\mathrm{H}}$ of this correlation provides an estimate of the radiation field intensity. For a set of clouds in Chamaleon with an opacity comparable to L1721 Boulanger et al. (1998) found a $I_{100 \mu \mathrm{m}} / N_{\mathrm{H}}$ ratio of $7.5 \pm 2.5 \mathrm{MJy} / \mathrm{sr} / \mathrm{mag}$ which corresponds to $4 \pm 1.3 \mathrm{MJy} / \mathrm{sr} /\left(10^{21} \mathrm{~cm}^{-2}\right)$. This study provides the reference value for the mean Solar Neighbourhood ISRF (Interstellar Standard Radiation Field). In L1721 


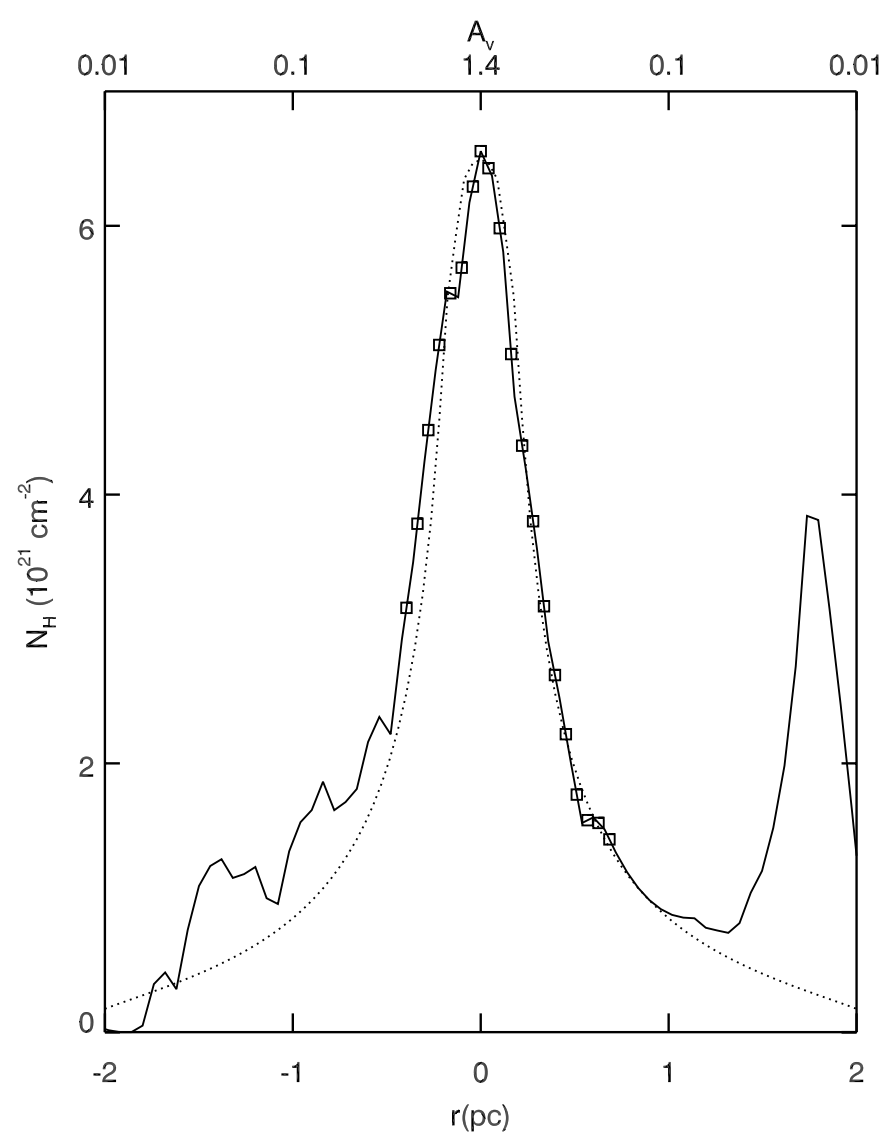

Fig. 5. Comparison of the column density observed (solid line) across L1721 and the analytic function fitted to it (dotted line) as a function of radial distance, $r$, and the visual extinction integrated from the cloud border, $A_{v}$. The peak observed at the right end of the cut is due to the plate saturation by the $\nu$ Sco star. The LWS positions are marked by squares.

we find a slope of $15 \pm 4$ for the cloud side opposite to $\nu$ Sco and $23 \pm 4$ for the cloud side facing $\nu$ Sco (see Fig. 6) which corresponds to 4 and 6 times the ISRF respectively. We emphasize here that the IRAS $100 \mu \mathrm{m}$ emission profile is relatively insensitive to abundance variations of the small grains (PAHs and VSGs) as shown in Bernard et al. (1992).

The radiation field heating L1721 is the sum of two contributions. The first contribution is from the $\nu$ Sco star, which has a luminosity of $6300 L_{\odot}$ and an effective temperature of $20000 \mathrm{~K}$. The contribution of $\nu$ Sco depends on the position of the star along the line of sight: we reference this position with an angle $\theta$ between the sky plane and the line connecting the cloud center to $\nu$ Sco (see Fig. 7). Next, the ISRF at this position is likely to be enhanced relative to the mean Solar Neighbourhood ISRF because L1721 is heated by the B and A stars cluster of Upper Scorpius. The Upper Scorpius complex contains a large proportion of stars with effective temperature of less than $20000 \mathrm{~K}$ (de Geus et al. 1989). We use the Mathis et al. (1983) radiation field to represent the mean Upper Scorpius radiation field. This last radiation field is assumed to be isotropic and in the following we call this

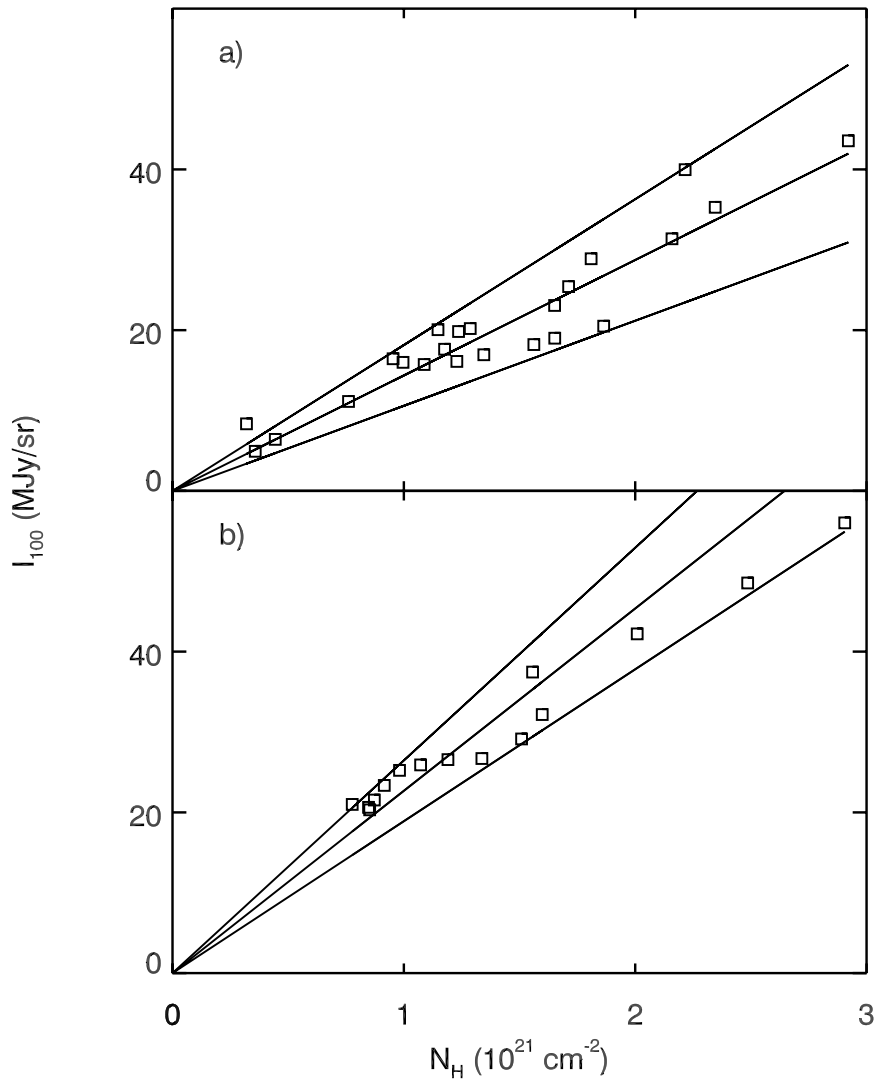

Fig. 6. The $100 \mu \mathrm{m}$ IRAS surface brightness a) opposite to $\nu$ Sco and $\mathbf{b}$ ) facing $\nu$ Sco as a function of the column density observed along each line of sight.

contribution the isotropic radiation field. The radiation field exciting L1721 thus only depends on the distance of $\nu$ Sco to the cloud center and on the strength of the Upper Scorpius contribution. The slope of the $100 \mu \mathrm{m}-N_{\mathrm{H}}$ correlation measures the power absorbed by Big Grains per hydrogen atom. This power depends on the intensity but also on the spectral distribution of the radiation field. In practice, for a given radiation field intensity at $1000 \AA$, the emission of BGs per $\mathrm{H}$ atom is 2.5 times larger for the Mathis et al. (1983) radiation field than for a $20000 \mathrm{~K}$ blackbody. We have taken into account this dependence to translate the $100 \mu \mathrm{m} / N_{\mathrm{H}}$ slopes into $\chi$-values which represent the strength of a radiation field at $\lambda=1000 \AA$ in units of $1.6 \times 10^{-3} \mathrm{ergs} / \mathrm{cm}^{2} / \mathrm{sr}$ (Habing 1968). The radiation field intensity is described in this way because the far-UV photons are more efficient at heating the gas. The strength of the $\nu$ Sco radiation field is represented by $\chi_{\text {sco }}$ and the strength of the isotropic radiation field by the factor $\chi_{\mathrm{I}}$. We have considered two cases:

1. $\chi_{\mathrm{I}}=1$ for the isotropic radiation field and $\chi_{\mathrm{sco}}=12.5$. In that case most of the radiation field strength on both cloud sides is due to $\nu \mathrm{Sco}$;

2. The Upper Scorpius isotropic radiation field on L1721 is the dominant radiation field on the cloud side opposite to $\nu$ Sco and then $\chi_{\mathrm{I}}=4$ and $\chi_{\text {sco }}=5$. 


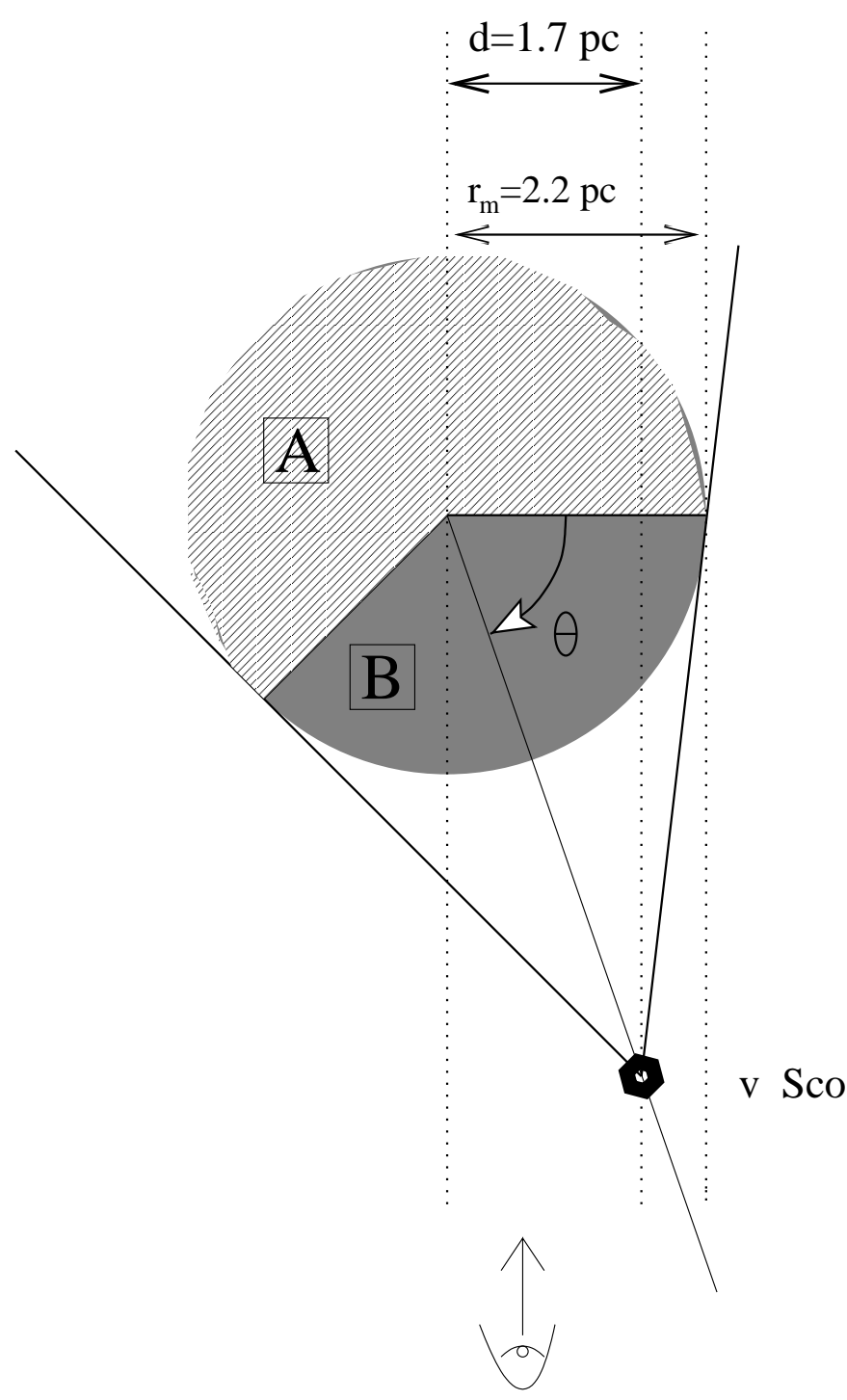

Fig. 7. Geometry used for modelling L1721. The projected distance of $\nu$ Sco to the cloud center is $1.7 \mathrm{pc}$.

A detailed modelling of the dust IR emission in L1721, based on the model of Bernard et al. (1992), is currently underway in our group to discriminate between these two cases. We expect that the case 2 radiation field will propagate more efficiently than the UV light towards the cloud center because of the strong visible component of the radiation field. Also, note that the effects of clumpiness can affect the penetration of star light inside the cloud. For the line modelling, we select the case 2 to describe the exciting radiation field of L1721. In Sect. 3.4, we will discuss the sensitivity of the gas cooling lines emission to the incident radiation field. In the case 2 , the contribution of $\nu$ Sco amounts to a mean value of $\chi_{\mathrm{sco}}=5$ for the cloud side facing $\nu$ Sco, which corresponds to $\theta=70^{\circ}$ for the position of the star. As the far-infrared extinction is small, a very similar $100 \mu \mathrm{m}$ emission band profile is expected for $\theta=-70^{\circ}$. We will see in Sect. 3.4 that $\theta=+70^{\circ}$ is favoured.

\subsection{PDR model}

To interpret the gas line emission we make use of the model described in Le Bourlot et al. (1993). In this model a PDR is represented by a semi-infinite plane-parallel slab with an isotropic radiation field incident on the interface. The input parameters are (i) $\chi$, the scaling factor for the radiation field, and (ii) the density profile. With these inputs the model solves the chemical and thermal balances starting from the slab edge. The scattering of the radiation by dust grains is treated with the formalism of Flannery et al. (1980) in the plane-parallel case and assumes a mostly forward scattering $(g=<\cos \alpha>=0.6$, where $\alpha$ is the scattering angle). For extinction properties, we use the analytical fit of Fitzpatrick \& Massa (1988) to the Galactic average extinction curve. The radiative transfer in the absorption lines of $\mathrm{H}_{2}$ and $\mathrm{CO}$ is treated in detail and the individual line profiles are treated with the prescription of Federman et al. (1979). We use recent gas phase elemental abundances measured in the diffuse interstellar medium: $\mathrm{He} / \mathrm{H}=0.1, \mathrm{C} / \mathrm{H}=1.4 \times 10^{-4}$ (Cardelli et al. 1996), and $\mathrm{O} / \mathrm{H}=3.19 \times 10^{-4}$ (Meyer et al. 1998).

For the photoelectric effect on small dust grains, we adopt the formalism of BT. In particular, our model takes into account the actual spectral distribution of the radiation field to compute the heating rate (Eqs. (1) and (14) of BT). The exponent of the power law size distribution of small grains is -3.5 while the lower and upper limits of the grain radius are 4 and $100 \AA$ respectively. Larger grains are not included in the computation of the photoelectric heating rate. They have a low photoelectric efficiency due to the large number of collisions the electron must undergo before escaping the grain (Verstraete et al. 1990). The computation of the photoelectric heating assumes that the small grains are graphitic and spherical. Grains with less than $50 \mathrm{C}$ atoms are likely to be planar: these species are less charged and consequently have a higher photoelectric efficiency (BT). However, for a given radiation field, the gain does not exceed $25 \%$ (Wolfire et al. 1995): such variations fall within our error bars on the gas cooling lines.

\subsection{Spatial distribution of line emission}

We now present results of the PDR model for the parameters described in the last three sections. The total strength, $\chi_{\mathrm{t}}=\chi_{\mathrm{I}}+\chi_{\mathrm{sco}}$, of the radiation field on the cloud surface ranges from 4 (opposite to $\nu$ Sco) to 11 (facing $\nu$ Sco). Figures 8 and 9 describe the chemical stratification and the thermal budget of the plane-parallel model as a function of depth into the cloud. In these figures we have used $\chi=\chi_{\mathrm{t}}=5$, which corresponds to the mean radiation field received by a particle located on the cloud surface facing $\nu$ Sco. We note that the photoelectric effect dominates by far the gas heat budget.

We now use the PDR model to compute profiles of line fluxes across the cloud which can be compared to the observations. To do this, we approximate the spherical shape 


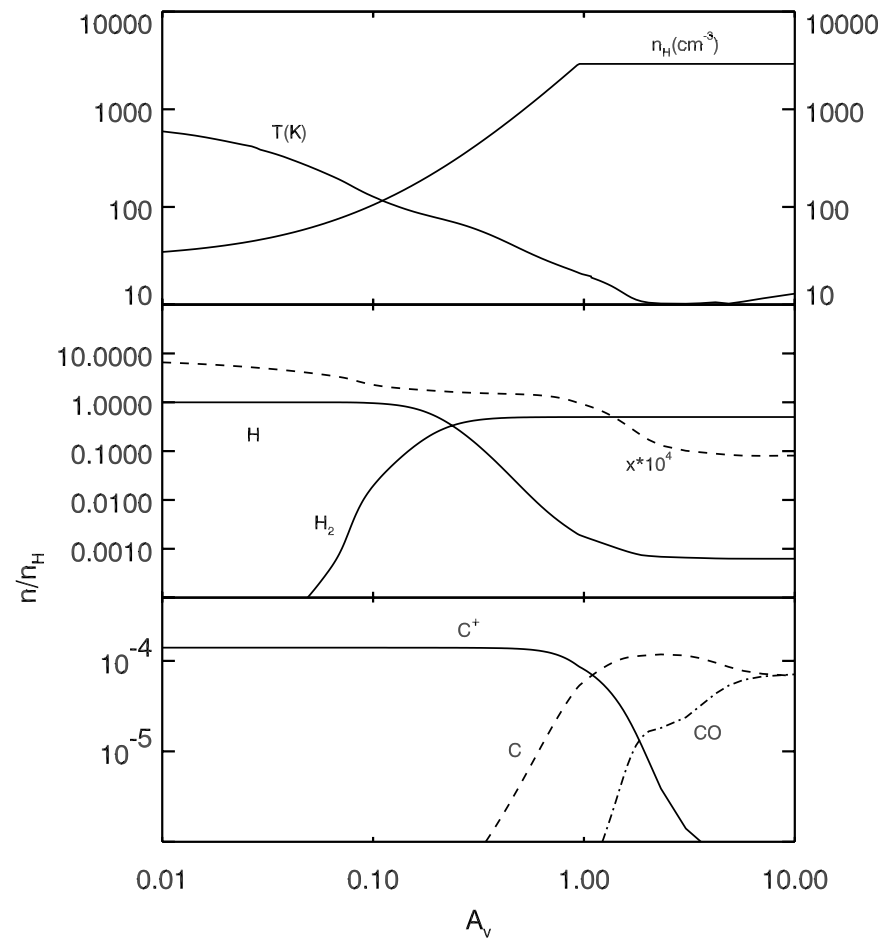

Fig. 8. Variation of the gas temperature, the gas density and the fractional ionization $x=n_{\mathrm{e}} / n_{\mathrm{H}}$ for a plane-parallel PDR model with $\chi=5$ and the density profile of Sect. 3.1. We also show the $\mathrm{H} / \mathrm{H}_{2}$ and $\mathrm{C}^{+} / \mathrm{C}^{0} / \mathrm{CO}$ transitions for the same model. The $\nu$ Sco star lies to the left end of the figure.

of the cloud by a combination of plane-parallel models. This allows us to take into account the variation of the radiation field at the cloud surface and the fact that each line of sight crosses diffuse, warm, interface gas as well as more deeply embedded, colder regions. We thus divide the cloud into angular sectors. The first sector takes up all the cloud side opposite to $\nu$ Sco (the area noted $A$ in Fig. 7). In addition, six equal sectors are defined on the side facing $\nu$ Sco (the area noted $B$ in Fig. 7 ): these sectors are symmetrically distributed with respect to the line connecting $\nu$ Sco to the cloud center. To each angular sector is associated one value of $\chi_{\mathrm{t}}$. The three sectors facing $\nu$ Sco on one side of the $\nu$ Sco-cloud center line have $\chi_{\mathrm{t}}=11,10$ and 9 (going away from the $\nu$ Sco-cloud center line). The large sector $A$ opposite to $\nu$ Sco receives $\chi_{\mathrm{t}}=\chi_{\mathrm{I}}=4$. We then run a PDR model for each sector with the density distribution of Sect. 3.1 and the corresponding value of $\chi_{\mathrm{t}}$. The model output is a local line emission rate $\left(J_{\nu}\right)$ which only depends on the optical depth measured along a radius from the cloud edge.

The line intensity integrated along a given line of sight is written as (see Fig. 10):

$I_{\nu}=\sum_{k, i} \frac{\Lambda_{\nu}(k, i)}{4 \pi} \times l(k, i)$.

In this expression, $k$ labels the angular sectors and $i$ the various layers (defined by the radial $A_{v}$ taken from the cloud edge) crossed by the line of sight within an angular

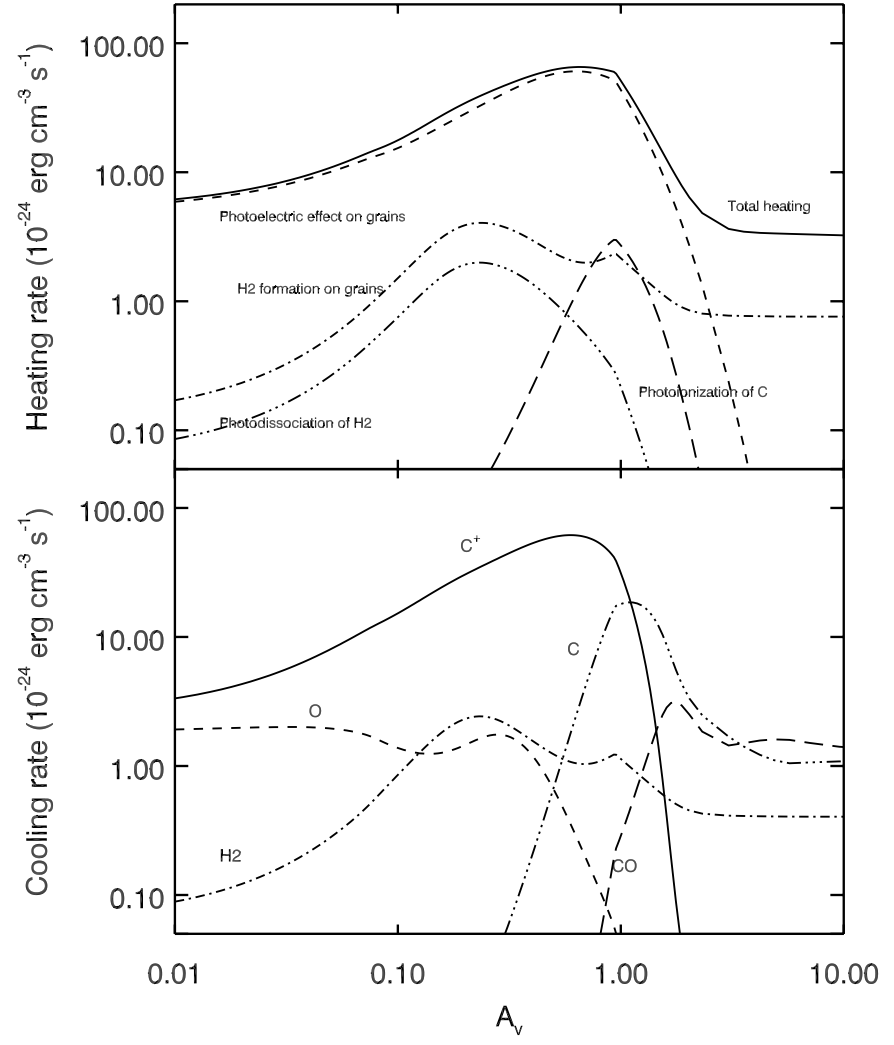

Fig. 9. Main heating and cooling rates for the PDR model $(\chi=5)$.

sector; $l(k, i)$ is the length in $\mathrm{cm}$ of the layer $i$ in the $k$ angular sector on the line of sight. The line cooling rate in $\mathrm{erg} / \mathrm{s} / \mathrm{cm}^{3} \Lambda_{\nu}(k, i)$ is equal to $J_{\nu}(k, i) \times \beta(i)$ where $J_{\nu}(k, i)$ is the line emissivity extracted from the PDR model and $\beta(i)$ the escape probability from layer $i$ to the cloud edge along the line of sight. For $\beta$, we use the formalism of Tielens \& Hollenbach (1985) in their Appendix B with a turbulent Doppler width $\delta v_{\mathrm{d}}=1 \mathrm{~km} \mathrm{~s}^{-1}$. We find that the $\left[\mathrm{C}^{+}\right] 158 \mu \mathrm{m}$ and $\left[\mathrm{O}^{0}\right] 63 \mu \mathrm{m}$ lines reach an optical depth of 1 at $A_{v}=0.5$ and 0.3 respectively. The $\mathrm{H}_{2}$ rotational lines are optically thin.

The model line intensity profiles results are compared to the data in Fig. 11. We have put the star at $\theta=+70^{\circ}$ (Fig. 7). Actually, for $\theta=-70^{\circ}$ the model line emission is significantly lower due to line opacity. With a dustto-gas mass ratio of the small grain populations (PAHs and VSGs) constant throughout the cloud and equal to $8.610^{-4}$, the observed $\mathrm{C}^{+}$and $\mathrm{O}^{0}$ line intensity profiles are not reproduced by the PDR model. Indeed, the $\left[\mathrm{C}^{+}\right] 158 \mu \mathrm{m}$ and $\left[\mathrm{O}^{0}\right] 63 \mu \mathrm{m}$ line intensities are underestimated by a factor of about 2 and 3 respectively. The uncertainty on the radiation field, mentionned in Sect. 3.2, amounts to relative error of $40 \%$ for $\mathrm{C}^{+}$and $70 \%$ for $\mathrm{O}^{0}$. Such error bars cannot explain the discrepancy between the observed and predicted profiles. Also a higher $\mathrm{C}$ and $\mathrm{O}$ abundances cannot accommodated this difference, because the fine structure line intensities are practically linearly dependant on the gas phase elemental abundances. Such discrepancies may arise from transfer effects on the 


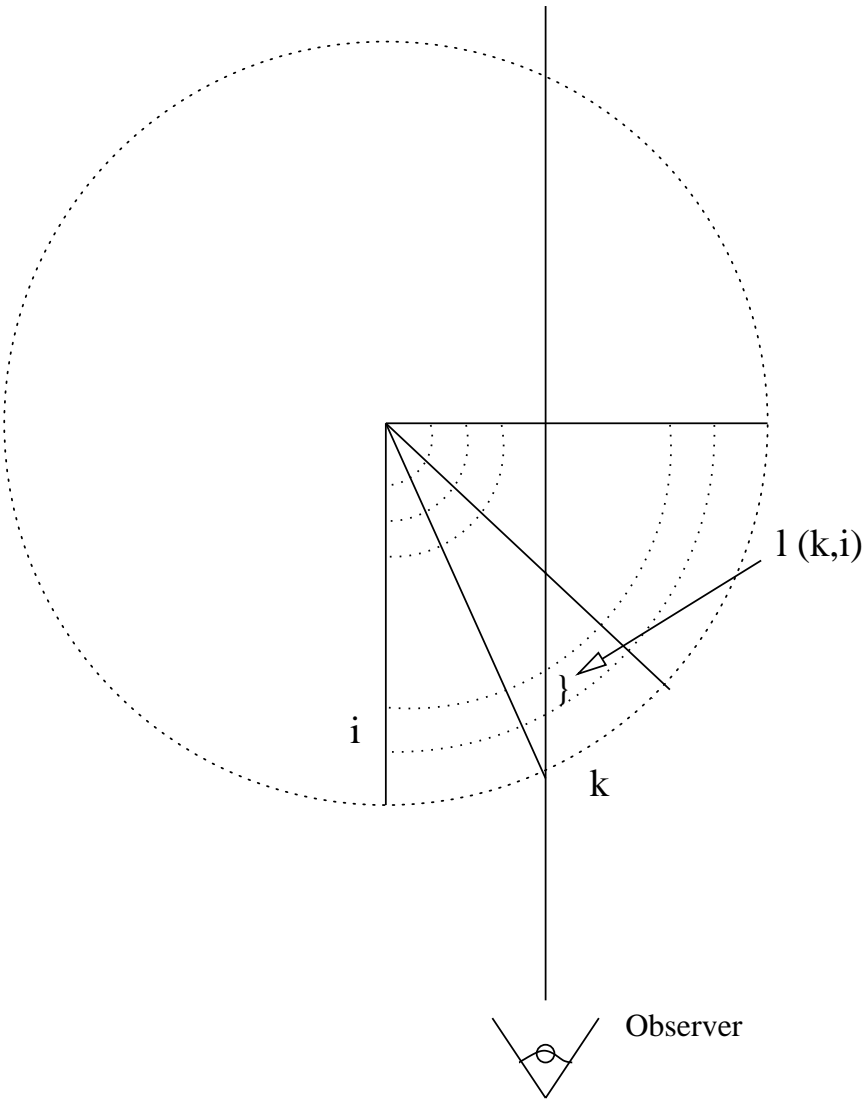

Fig. 10. Geometry used to calculate the line emission profiles intensities. Index $k$ labels the various angular sectors while $i$ labels the $A_{v}$-layers of the PDR models.

emerging cooling lines and/or an underestimated heating rate.

In the PDR model, the non-thermal velocity fields are represented by the turbulent Doppler width $\delta v_{\mathrm{d}}$ which is added to the thermal width (Tielens \& Hollenbach 1985). This parameter affects the transfer of resonant UV photons through the absorption lines of $\mathrm{H}_{2}$ and $\mathrm{CO}$ and the opacity of the emerging IR cooling lines. We show the result of varying $\delta v_{\mathrm{d}}$ in Fig. 11: for a reasonable range of $\delta v_{\mathrm{d}}$-values, we see that decreasing the opacity of the emerging cooling lies does not account for the observed intensities, in particular $\left[\mathrm{O}^{0}\right]$.

Alternatively, the low intensity of the gas cooling lines in the model may tell us that the gas temperature is too low (see Fig. 8). Indeed, the predicted $\left[\mathrm{O}^{0}\right] 63 \mu \mathrm{m}$ line emission is very sensitive to the gas temperature because the energy of the transition corresponds to a temperature of $228 \mathrm{~K}$. Thus, another solution to match the data is to increase the heating rate. This latter scales with the UV radiation field intensity $(\chi)$, the small grains abundance and the photoelectric efficiency (BT). The band ratios shown in Fig. 12 suggest that the PAHs and VSGs abundance vary across the cloud. In the next subsection we estimate these variations and show that they have an important impact on the emerging gas cooling. We have not attempted to change the abundance of BGs, because

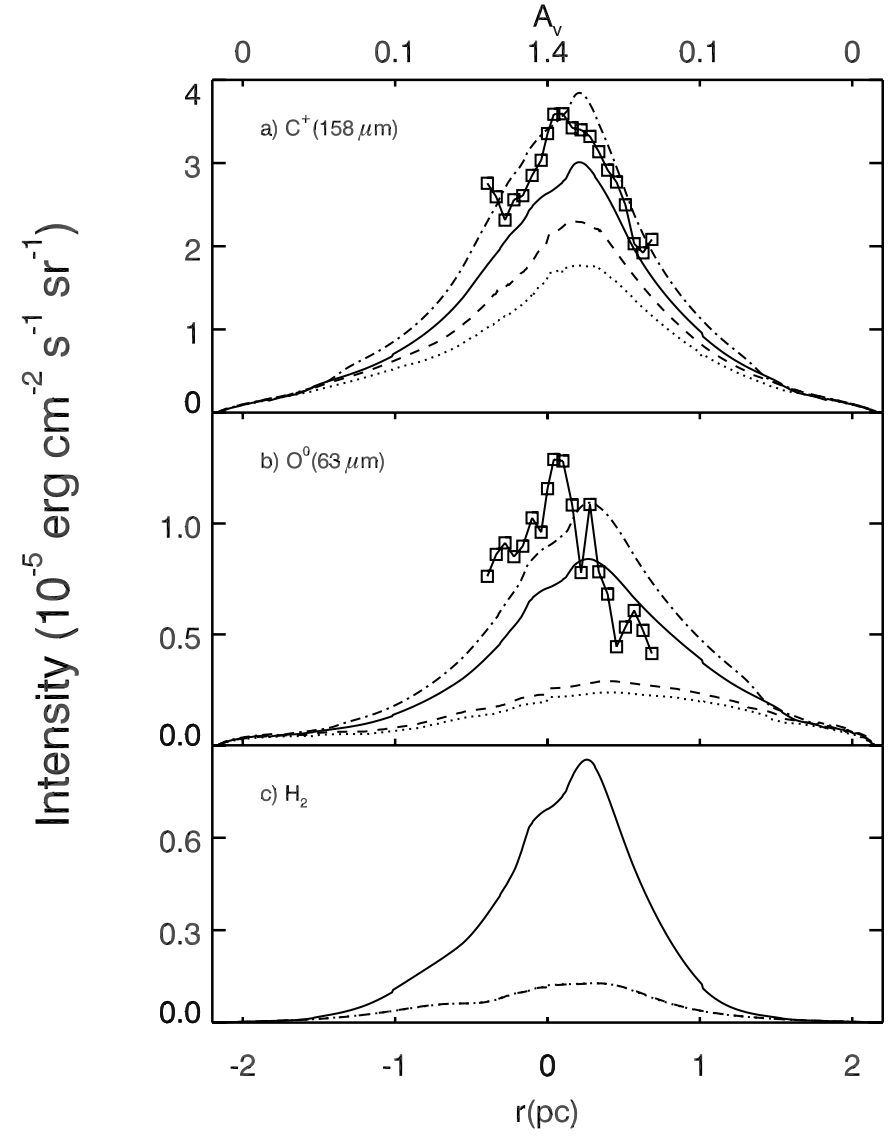

Fig. 11. Line emission profiles as observed (solid lines with squares) and predicted by the PDR model as a function of radius and of visual extinction from the cloud edge. The dotted and dashed lines correspond to a calculation with a small dust grain abundance constant throughout the cloud and $\delta v_{\mathrm{d}}$ equal to $1 \mathrm{~km} \mathrm{~s}^{-1}$ and $3 \mathrm{~km} \mathrm{~s}^{-1}$ respectively. The model with small dust grain abundance variations and $\delta v_{\mathrm{d}}=1 \mathrm{~km} \mathrm{~s}^{-1}$ or $3 \mathrm{~km} \mathrm{~s}^{-1}$ is represented by the solid and dot-dash lines respectively. The $\mathrm{H}_{2}$ line cooling has not been observed.

as mentioned in the Sect. 3.3, these large grains have a low photoelectric efficiency.

\subsection{Abundance variations of the small grains}

In this section, we first use the dust brightness profile to quantify the abundance variations of small dust grains. We then include these abundance variations in the PDR model and estimate the corresponding line intensity profiles as in the last section. Here we test the idea that the discrepancy between the observed and the predicted profiles is due to abundance variations of the small dust grains. Ratios of the brightness profiles of Fig. 4 are presented in Fig. 12.

We have estimated the abundance variations of small grains from the ratio of PAHs and VSGs emission to that of BGs. A coherent modelling of the dust emission from a spherical cloud (e.g. Bernard et al. 1992, 1993) which includes consistent dust abundance variations is beyond the scope of the present paper. We estimate the model IR emission of dust grains $I_{\mathrm{PAH}}^{\mathrm{mod}}, I_{\mathrm{VSG}}^{\mathrm{mod}}$ and $I_{\mathrm{BG}}^{\mathrm{mod}}$ as 


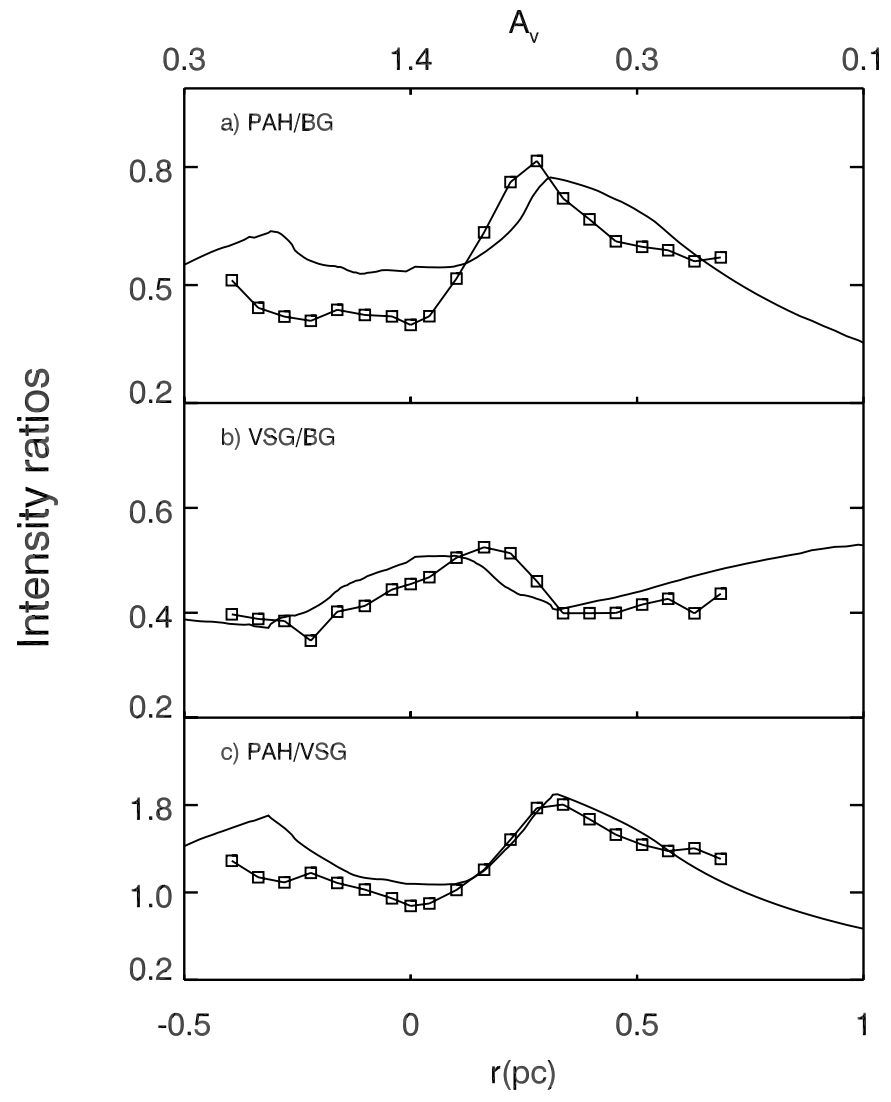

Fig. 12. Emission ratios of the different dust components as observed (solid lines with squares) and as predicted (solid lines) as a function of radius and of visual extinction from the cloud edge.

follows. The power in the IR emission of the different dust components, $X=[\mathrm{PAH}, \mathrm{VSG}, \mathrm{BG}]$, is equal to the power absorbed, i.e.:

$I_{\mathrm{X}}^{\mathrm{mod}}=\left[\frac{X}{H}\right] \times \sum_{k} \sum_{i} P_{\mathrm{abs}}^{\mathrm{X}}(k, i) \times \frac{n_{\mathrm{H}}(k, i)}{4 \pi} \times l(k, i)$

where

$P_{\mathrm{abs}}^{\mathrm{X}}(k, i)=\int_{0}^{13.6 \mathrm{eV}} \sigma_{\mathrm{X}}(E) F_{k, i}(E) \mathrm{d} E$

is the power absorbed in erg s $\mathrm{s}^{-1} \mathrm{H}^{-1}$ with $\sigma_{\mathrm{X}}$, the absorption cross-section per grain for PAHs, VSGs and BGs. $F_{k, i}(E)$ is the radiation field (in $\operatorname{erg~} \mathrm{s}^{-1} \mathrm{~cm}^{-2} \mathrm{eV}^{-1}$ ), found from the transfer prescriptions described in Sect. 3.3, and $n_{\mathrm{H}}(k, i)$ the density in each cloud zone characterised by the index $k$ and $i$ (see Fig. 10). For the BGs, we take the cross section of Désert et al. (1990), for PAHs we use Verstraete \& Léger (1992) and Draine \& Lee (1984) for VSGs. In the ISRF, PAHs absorb $2.3 \times 10^{-27}$ Watt $/ \mathrm{C}$ whereas graphitic VSGs only absorb $1.7 \times 10^{-27}$ Watt/C. Formula (4) can then be inverted to infer the dust abundance $(\mathrm{C} / \mathrm{H}$ in PAHs and VSGs) as a function of cloud radius. The resulting abundance profiles are presented in Figs. 11-13. The abundance of PAHs has to be multiplied by $\sim 5$ in the $\left[0.25<A_{v} \leq 0.45\right]$-layer to account for the

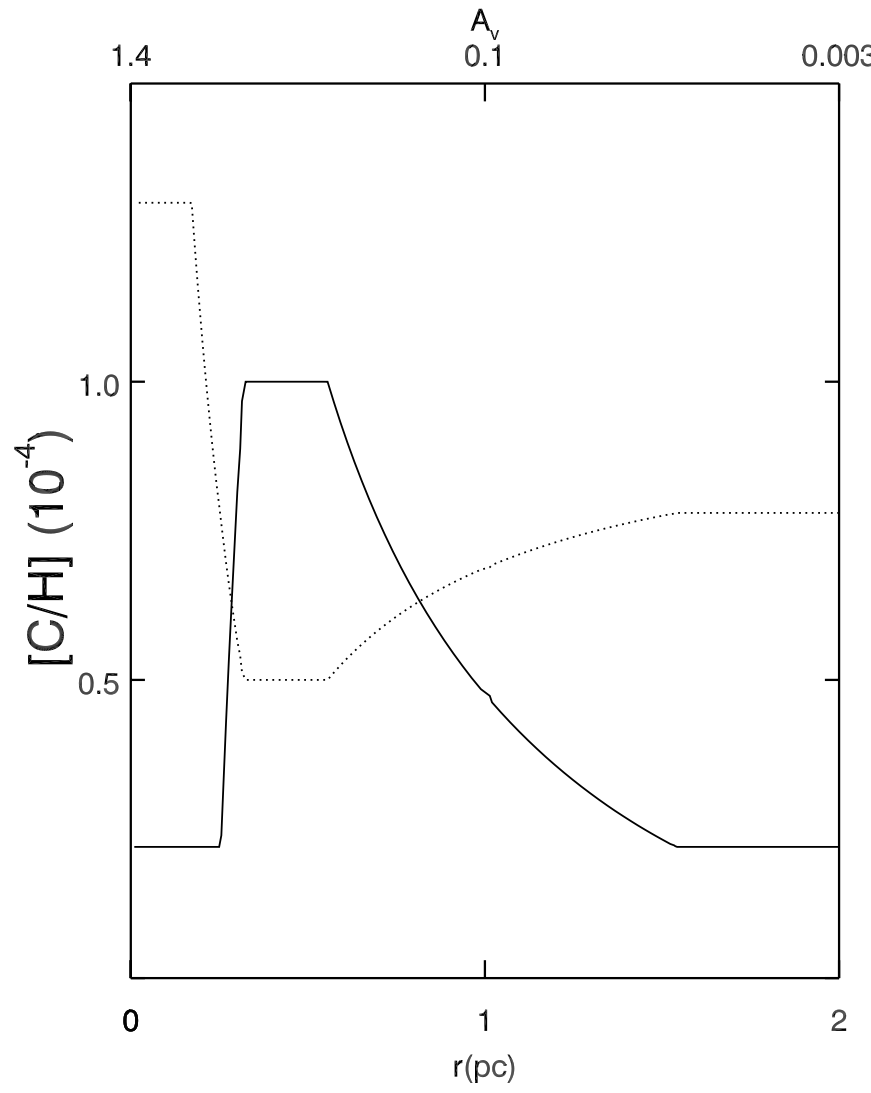

Fig. 13. Variation of the interstellar carbon abundance in PAHs (solid line) and VSGs (dotted line) of PDR models as a function of radius and of visual extinction integrated from the cloud border.

observed $I_{\mathrm{PAH}} / I_{\mathrm{BG}}$ ratio. Similarly, the VSGs abundance must be multiplied by $\sim 2$ in the $\left[A_{v} \geq 0.75\right]$-layer in order to reproduce the observed $I_{\mathrm{VSG}} / I_{\mathrm{BG}}$ emission ratio.

Including these abundance variations in the PDR modelling, we obtain the result displayed in Fig. 11. The corresponding line intensities are higher: the $\mathrm{C}^{+}$and $\mathrm{O}^{0}$ lines emission are increased by a factor about 1.5 and 3 respectively. The discrepancy at the center of the cloud between the observed and predicted line profiles may result from uncertainties in the radiation field, the radiation transfer, or the photoelectric efficiency and from our rough estimate of the small dust grain abundance profiles. In the present PDR modelling we have not attempted to include changes in the UV extinction curve associated with the small dust grain abundance variations because recent observations (Boulanger et al. 1994) have questioned the contribution of small dust grains to the extinction curve adopted in the current dust models (e.g. Désert et al. 1990; Dwek et al. 1997). However, exploring a range of far-UV to visible extinction ratio (from 5 to 10 at $1000 \AA$ ) we find the cooling lines to vary by less than $50 \%$. We therefore conclude that the observed intensity profile of the main gas cooling lines can only be reproduced if the abundance of PAHs is significantly increased. This result illustrates the dominant role of the smallest dust grains in the gas heating. It also shows that the model photoelectric 
Table 1. Average power radiated per hydrogen atom by gas coolants and small dust grains towards L1721.

\begin{tabular}{|c|c|c|c|}
\hline Species & Power $^{a}$ : & Observed & Theoretical \\
\hline$\left[\mathrm{e}^{+}\right] 158 \mu \mathrm{m}$ & & 1.2 & 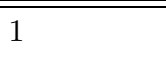 \\
\hline$\left[\mathrm{O}^{0}\right] 63 \mu \mathrm{m}$ & & 0.34 & 0.32 \\
\hline $\mathrm{H}_{2}$ & & & 0.27 \\
\hline Total gas cooling & & $1.81^{b}$ & 1.59 \\
\hline$I_{\mathrm{PAH}}+I_{\mathrm{VSG}}$ & & 78 & 54 \\
\hline$\epsilon_{\mathrm{PE}}$ & & 0.023 & 0.029 \\
\hline
\end{tabular}

${ }^{a} \operatorname{In~} 10^{-25} \mathrm{erg} \mathrm{s}^{-1} \mathrm{H}^{-1}$.

${ }^{b}$ Including the $\mathrm{H}_{2}$ contribution as estimated from the model.

efficiency is close to its actual value. In Table 1 , the values of the power radiated by gas coolants and small dust grains as observed and modelled are listed.

\section{Dust photoelectric efficiency}

Using our model estimate of the $\mathrm{H}_{2}$ cooling, we can now estimate the photoelectric efficiency by comparing the observed dust and gas emission. A relationship is theoretically expected between the main cooling rates and the dust emission. Indeed, a dust grain exposed to a stellar radiation field dissipates the energy it absorbs mostly as IR emission. A small fraction, $\epsilon$, of the energy absorbed is channelled to the gas by the photoelectric effect. The heating efficiency of this latter process is defined as

$\epsilon=\frac{P_{\mathrm{PE}}}{P_{\mathrm{abs}}}$

where $P_{\text {abs }}$ is the total power absorbed by the dust grains and $P_{\mathrm{PE}}$ is the power given to the gas through the photoelectric effect. For a gas in thermal balance, the gas heating and cooling rates are equal. In the L1721 PDR, the gas heating is dominated by the photoelectric effect. Assuming thermal balance the photoelectric heating is thus roughly equal to the total cooling rate. As long as the opacity of the emerging cooling lines is small, we can replace $P_{\mathrm{PE}}$ by the total gas cooling line emissions $I_{\Lambda}$. Moreover, as the power conveyed by the photoelectric effect is much smaller than the absorbed power $\left(\epsilon \sim\right.$ a few $\left.10^{-2}\right)$, the latter power is well approximated by the dust IR emission. Finally, $\epsilon$ can be observationally defined as:

$\epsilon \simeq \frac{I_{\Lambda}}{I_{\mathrm{dust}}}$

where $I_{\text {dust }}$ is the total dust IR emission.

As the photoelectric heating rate is expected to be dominated by the small grain populations, it is interesting to determine the photoelectric efficiency for each grain population. Along the LWS cut, we define:

$I_{\Lambda}^{\mathrm{d}}=\epsilon_{\mathrm{PAH}} I_{\mathrm{PAH}}+\epsilon_{\mathrm{VSG}} I_{\mathrm{VSG}}+\epsilon_{\mathrm{BG}} I_{\mathrm{BG}}$

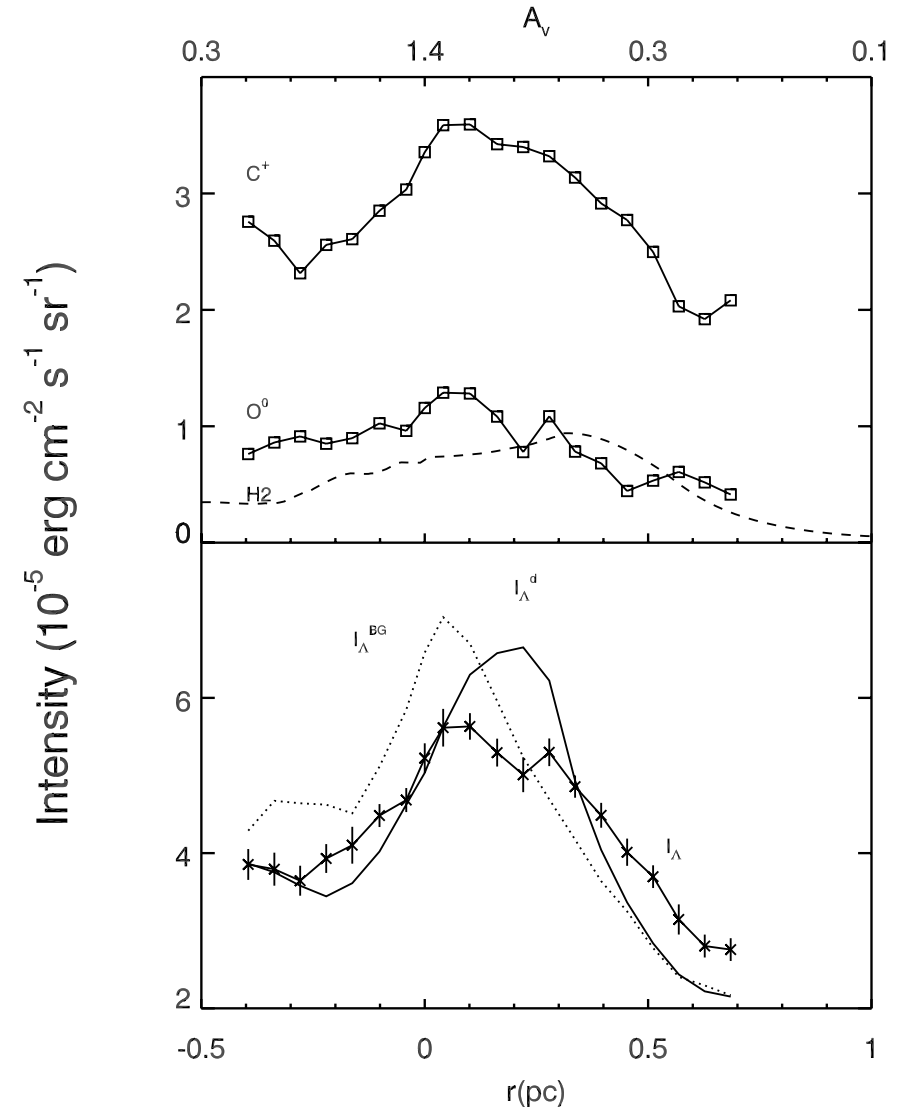

Fig. 14. Upper panel: intensities in the $\mathrm{C}^{+}$and $\mathrm{O}^{0}$ lines measured by LWS, and cooling in the $\mathrm{H}_{2}$ rotational lines (see Sect. 3.4). Lower panel: the total gas cooling, $I_{\Lambda}=$ $I_{\mathrm{C}+}+I_{\mathrm{O}^{0}}+I_{\mathrm{H}_{2}}$ with $3 \sigma$ error bars (solid line with crosses) and as derived from the dust IR emission: (i) $I_{\Lambda}^{\mathrm{d}}=0.03 \times$ $I_{\mathrm{PAH}}+0.01 \times I_{\mathrm{VSG}}+0.001 \times I_{\mathrm{BG}}$ (solid line) and (ii) $I_{\Lambda}^{\mathrm{BG}}=$ $0.001 \times I_{\mathrm{PAH}}+0.001 \times I_{\mathrm{VSG}}+0.02 \times I_{\mathrm{BG}}$ (dotted line).

with $\epsilon_{\mathrm{PAH}}, \epsilon_{\mathrm{VSG}}$ and $\epsilon_{\mathrm{BG}}$ the photoelectric efficiencies of the PAHs, VSGs and BGs respectively and $I_{\mathrm{PAH}}, I_{\mathrm{VSG}}$ and $I_{\mathrm{BG}}$ the corresponding dust emission. The photoelectric efficiencies can then be found from a least-square fit of $I_{\Lambda}^{\mathrm{d}}$ to $I_{\Lambda}$. In this derivation, we assume a constant photoelectric efficiency for each dust component across the cloud: as we show below (see Fig. 15), this is a reasonable approximation. From our best fit, we find that the small grain populations have a high photoelectric efficiency, namely $\epsilon_{\mathrm{PAH}}=3 \%, \epsilon_{\mathrm{VSG}}=1 \%$ and $\epsilon_{\mathrm{BG}}=0.1 \%$ (see Fig. 14). This combination reproduces well the observed gas emission in the outer parts of the cloud, but there is a significant deviation in the central region of the cloud. In fact, as discussed in Sect. 3.4, the gas cooling lines become optically thick towards the cloud center whereas the dust emission remains optically thin throughout the cloud: this is probably why $I_{\Lambda}^{\mathrm{d}}$ clearly overestimates $I_{\Lambda}$ at $r \sim 0.2 \mathrm{pc}$. We also show in Fig. 14 the opposite case where the BGs dominate the photoelectric heating rate: the corresponding cooling emission $I_{\Lambda}^{\mathrm{BG}}$ is not a good representation of $I_{\Lambda}$ along the whole LWS cut. Our results clearly show that the dust photoelectric heating is dominated by the smaller 
grains, and not by the BGs. However, the spatial correlation between dust and gas emission does not allow us to tightly constrain the photoelectric efficiency of the various grain components because of the spherical geometry where outer and inner regions contribute to the emergent intensity at all sky positions.

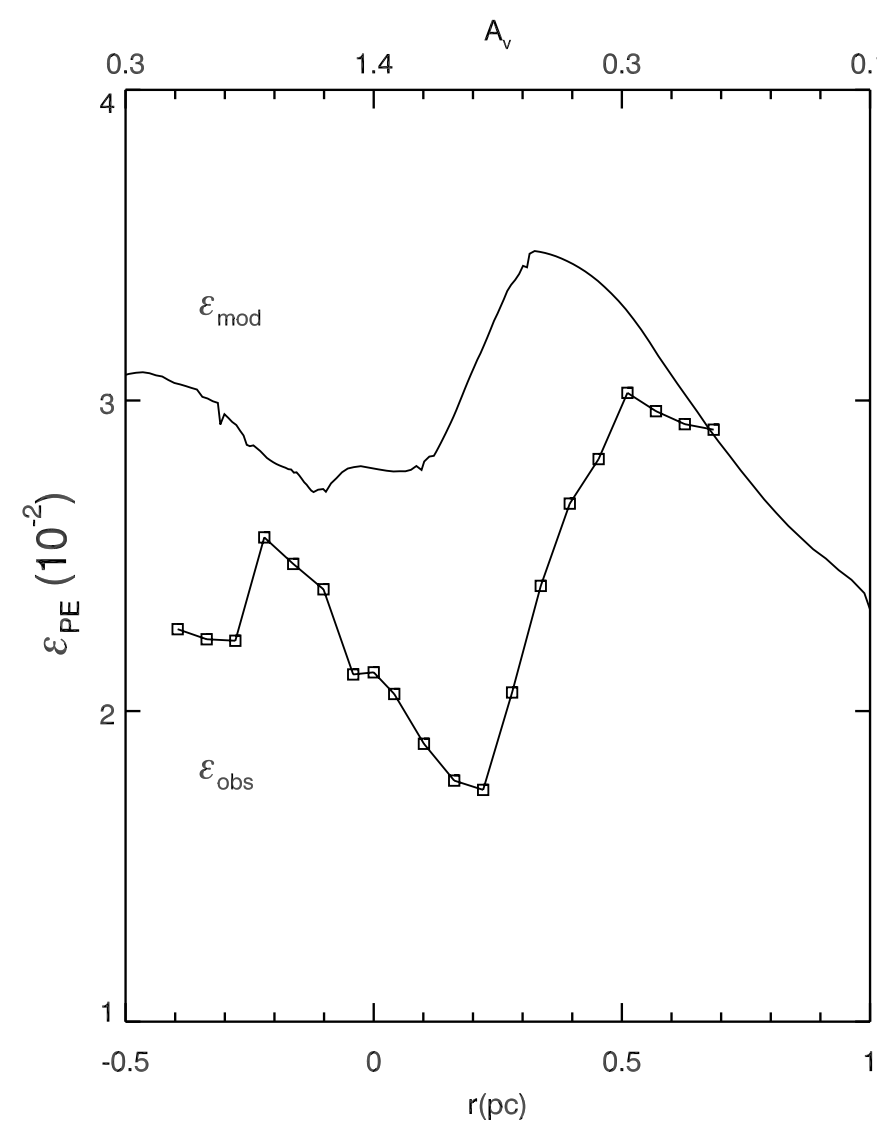

Fig. 15. Comparison between the theoretical efficiency $\epsilon_{\bmod }$ (solid line and see text) and our observational value $\epsilon_{\mathrm{obs}}=$ $I_{\Lambda} /\left(I_{\mathrm{PAH}}+I_{\mathrm{VSG}}\right)$ with $I_{\Lambda}=I_{\mathrm{C}^{+}}+I_{\mathrm{O}^{0}}+I_{\mathrm{H}_{2}}$ (solid line with squares) as a function of $r$ and $A_{v}$.

We can estimate the observational photoelectric efficiency profile of small grains across the cloud as $\epsilon_{\mathrm{obs}}=$ $I_{\Lambda} /\left(I_{\mathrm{PAH}}+I_{\mathrm{VSG}}\right)$. This efficiency is compared in Fig. 15 to the theoretical curve $\epsilon_{\bmod }$ which has been derived from our PDR modelling with the dust abundance variations discussed in Sect. 3.5. For consistency with the observations, we define $\epsilon_{\text {mod }}$ as the ratio between the emergent gas cooling lines and the power absorbed by small grains as computed in Sect. 3.5. We see in Fig. 15 that $\epsilon_{\bmod }$ reproduces the main features of $\epsilon_{\mathrm{obs}}$ across the cloud and that the $\epsilon_{\bmod }$-values are within $30 \%$ of those of $\epsilon_{\mathrm{obs}}$. The shape of the $\epsilon_{\bmod }$-profile can be understood as follows. While penetrating into the cloud $(r \geq 0.3 \mathrm{pc})$, the UV radiation is attenuated and the average grain charge (positive) decreases; consequently, $\epsilon_{\bmod }$ increases because of the weaker Coulomb barrier for the ejection of electrons from the grain (BT). Towards the center of the cloud $(r \leq 0.3 \mathrm{pc})$, the photoelectric efficiency decreases. This trend is the combination of two effects. On the one hand, the opacity of the cloud center to UV photons is larger than for visible photons: since the photoelectric effect is produced mostly by UV photons, $\epsilon_{\text {mod }}$ diminishes towards the cloud center. On the other hand, the $\epsilon_{\bmod }$-drop at $r \leq 0.3 \mathrm{pc}$ also reflects the behaviour of the $n_{\mathrm{PAH}} / n_{\mathrm{VSG}}$ ratio (see Fig. 12). Comparing $\epsilon_{\bmod }$ and $\epsilon_{\mathrm{obs}}$ in detail, we see that the efficiency values at the cloud boundary are quite comparable. Moving into the cloud, $\epsilon_{\mathrm{obs}}$ is systematically lower than $\epsilon_{\bmod }$. Also, the ridge and trough of the $\epsilon_{\bmod }$-profile are somewhat shifted with respect to $\epsilon_{\text {obs }}$. These discrepancies probably result from the simplified geometry and radiation transfer we adopted for the L1721 cloud, as well as the uncertainty in the spectral distribution of the exciting radiation field (see Sect. 3.2). In this latter case, a detailed modelling of the dust infrared emission is warranted in order to consistently derive the exciting radiation field and the dust abundance profiles in the L1721 cloud.

\section{Conclusion}

We study the gas thermal balance and the dust IR emission across L1721 a nearby, isolated molecular cloud heated by the $\nu$ Sco star and by the BA star association of Upper Scorpius. The cooling of the gas is obtained with ISO-LWS measurements of the major cooling lines, $\left[\mathrm{C}^{+}\right] 158 \mu \mathrm{m}$ and $\left[\mathrm{O}^{0}\right] 63 \mu \mathrm{m}$. The dust IR emission is traced with IRAS data.

We model the gas cooling lines with an updated version of the photodissociation region model of Le Bourlot et al. (1993). The input physical conditions in this modelling are the density profile and the exciting radiation field. The density profile of the L1721 cloud is deduced from visual extinction data $\left(A_{v}\right.$ at the center is $\sim 3$ ). The exciting radiation field is estimated to be $\chi=5$ to 10 times the radiation field of the Solar Neighbourhood from the ratio of dust emission to gas column density.

The spatial correlation between the gas cooling lines and the IR emission of small grains (PAHs and VSGs of radii $\leq 100 \AA$ ) confirms the theoretical expectation that the gas heat budget, in the less excited PDRs, is dominated by the photoelectric effect on small grains. The photoelectric efficiency measured from the ratio of the total gas cooling to the dust IR emission is in the range 2 to $3 \%$, in good agreement with current theoretical models (Bakes \& Tielens 1994; Weingartner \& Draine 2001).

Moreover, we find that the gas cooling lines of the L1721 cloud cannot be explained if the abundance of small grains is kept constant across the cloud, equal to the abundance of the diffuse ISM. Rather, as indicated by the IRAS band ratios, our PDR model results matches the gas cooling line observations when the abundance of PAHs is enhanced by a factor of 5 towards the cloud center. 
Acknowledgements. We are grateful to L. Cambrésy for providing us the extinction map of L1721. We also thank E. Caux and W. T. Reach for their help with the LWS data reduction.

\section{References}

Bakes, E. L. O., \& Tielens, A. G. G. M. 1994, ApJ, 427, 822

Bernard, J. P., Boulanger, F., Désert, F. X., \& Puget, J. L. 1992, A\&A, 263, 258

Bernard, J. P., Boulanger, F., \& Puget, J. L. 1993, A\&A, 277, 609

Bernard, J. P., Boulanger, F., Désert, F. X., et al. 1994, A\&A, 291, L5

Boulanger, F., Falgarone, E., Puget, J. L., \& Helou, G. 1990, ApJ, 364, 136

Boulanger, F., Prevot, M. L., \& Gry, C. 1994, A\&A, 284, 956

Boulanger, F., Abergel, A., Bernard, J. P., et al. 1996a, A\&A, 312,256

Boulanger, F., Reach, W. T., Abergel, A., et al. 1996b, A\&A, 315, L325

Boulanger, F., Bronfman, L., Dame, T. M., \& Thaddeus, P. 1998, A\&A, 332, 273

Cambrésy, L. 1999, A\&A, 345, 65

Cardelli, J. A., Meyer, D. M., Jura, M., \& Savage, B. D. 1996, ApJ, 467, 334

Caux, E., \& Gry, C. 1997, Iso lws spectroscopy of the diffuse interstellar medium, in The Far Infrared and Submillimetre Universe, 67

Clegg, P. E., Ade, P. A., Armand, C., Baluteau, J. P., \& Barlow, M. J., et al. 1996, A\&A, 315, L38

de Geus, E. J., DeZeeuw, P. T., \& Lub, J. 1989, A\&A, 216, 44

Désert, F. X., Boulanger, F., \& Puget, J. L. 1990, A\&A, 237, 215
Draine, B. T., \& Lee, H. M. 1984, ApJ, 285, 89

Dwek, E., Arendt, R. G., Fixsen, D. J., et al. 1997, ApJ, 475, 565

Federman, S. R., Glassgold, A. E., \& Kwan, J. 1979, ApJ, 227, 466

Fitzpatrick, E. L., \& Massa, D. 1988, ApJ, 328, 734

Flannery, B. P., Roberge, W., \& Rybicki, G. B. 1980, ApJ, 236,598

Habing, H. J. 1968, Bull. Astron. Inst. Netherlands, 19, 421

Hollenbach, D. J., \& Tielens, A. G. G. M. 1999, Rev. Mod. Phys., 71, 173

Hollenbach, D. J., Takahashi, T., \& Tielens, A. G. G. M. 1991, ApJ, 377, 192

Kemper, C., Spaans, M., Jansen, D. J., et al. 1999, ApJ, 515, 649

Kessler, M. F., Steinz, J. A., Anderegg, M. E., et al. 1996, A\&A, 315, L27

Le Bourlot, J., Pineau Des Forêts, G., Roueff, E., \& Flower, D. R. 1993, A\&A, 267, L233

Lynds, B. T. 1962, ApJS, 7, L1

Mathis, J. S., Mezger, P. G., \& Panagia, N. 1983, A\&A, 128, 212

Meyer, D. M., Jura, M., \& Cardelli, J. A. 1998, ApJ, 493, 22

Tielens, A. G. G. M., \& Hollenbach, D. 1985, ApJ, 291, 722

Verstraete, L., \& Léger, A. 1992, A\&A, 266, 513

Verstraete, L., Léger, A., D'Hendecourt, L., Defourneau, D., \& Dutuit, O. 1990, A\&A, 237, 36

Weingartner, J. C., \& Draine, B. T. 2001, submitted to ApJ, [astro-ph/9907251]

Wolfire, M. G., Hollenbach, D., McKee, C. F., Tielens, A. G. G. M., \& Bakes, E. L. O. 1995, ApJ, 443, 152

Wright, E. L., Mather, J. C., Bennett, C. L., Cheng, E. S., \& Shafer, R. A., et al. 1991, ApJ, 381, 200 